\title{
Does Health Care Need Non Profit Organizations? A Sample of a Home Palliative Care Service in Europe
}

\author{
Raffaella Pannuti* \\ ANT Italia Foundation, Italy
}

Received: February 13, 2014; Accepted: February 26, 2014; Published: February 28, 2014

*Corresponding author: Raffaella Pannuti, ANT Italia Foundation President, via Jacopo di Paolo 36, 40128 Bologna, Italy, Tel: $00390517190173 ;$ Fax: 0039051 7190150; E-mail: raffaella.pannuti@ant.it

\section{Letter to Editor}

The health and social costs of cancer are increasing. Health spending on cancer care is growing in light of the progress achieved in the field of diagnosis and treatment, leading to an increase of survival and higher economic impact of techniques. To this we must add the weight of the social costs related to oncological pathology, i.e., the set of tangible and intangible costs that cancer patients and their families are called to face. The need to find the necessary resources to ensure adequate cancer care is in contrast with the current economic crisis which is going throughout the European Union and more generally the Western world. This highlights the inadequacy of current institutions to sustain essential standards of care.

Nowadays, in a lot of European Countries a spending review of the costs of health care is taking place aimed at the demolition of waste and obvious inconsistencies, but also causing a substantial shortage of hospital beds. It's clear that this trend of health policies is necessary to provide the "shock absorbers" that can meet the needs of an aging population at a steady rate and for which it must provide in the future an expansion of investments. In this sense, the spending review cannot be understood only as an instrument of control over expenditure, but it's necessary to reconsider how to spend. It is therefore not only the "cutting", but the reviewing of the processes that will help obtaining lower costs and better services.

It's time to seriously rethink the assistance by integrating social and health support, in order to respond effectively not only to the current challenges, but especially to those of the future. For a truly sustainable health care it is desirable to adopt a view of public-private capital integration. We have to give up the idea of a "public health for few people" and lean towards a more integrated health care for everybody. If we do not think of going through the welfare state to welfare society, in which the service public assistance is helped by the free initiative of the citizens, we would never think to keep the universalism of services that is one of the most significant achievements of European civilization.

Within the European scene, palliative care is already a challenge, but definitely home care is the social innovation that health care needs and that we want to discuss. But the big challenge in front of us is the renewed relationship between doctor and patients, away from the mechanical nature of the performance, but close to dignity and quality of life of the person.

The field of palliative care shows more clearly than others the role played by non-profit organizations (NPOs) - precisely expression of civil society - actively engaged in this area of interest.

In recent decades, it's also thanks to the contribution of a private non profit organization that palliative care in Italy has been able to develop first in the field and later in a more structured way through a legislative formalization initiated by Law n. 38 of 2010. This Law intend to guarantee the access to palliative care and pain therapy for all patients, as ratified by the State-Regions agreement in July 2012.

36 years have passed since May 15, 1978, when we founded ANT Italia Foundation in Bologna with the intent of doing research and launching a project of home care for helping cancer patients and their families. In his daily work as the head oncologist at Sant'Orsola Malpighi Hospital, the ANT founder, was aware of the difficulties that he had every time when he had to dismiss patients to "free beds", always too scarce, to be given to other patients awaiting chemotherapy. He was in trouble because he wanted to help them even after treatment. Until the end, he understood that this was what they were asking him, and so were their families. Since then, ANT has assisted nearly 100,000 sufferers, of which 10,000 only in 2013. About 4,000 patients are assisted every day by more than 250 ANT healthcare professionals (physicians, psychologists, nurses and physiotherapists) with 20 offices located in 9 Italian regions, taking care of their patients 24 hours a day, 7 days a week, offering not only health care but also social and spiritual support.

The characteristic and strength of this charity organization is the ability to keep together all the various groups and organize them equally.

ANT Italia Foundation, as the biggest organization that takes care of palliative home care in Italy, constitutes a privileged observatory. We follow approximately $10 \%$ of cancer patients 
assisted at home in different Italian regions. Through a dedicated palliative care system it is possible to avoid inappropriate admissions and suffering to the patient and family members. This involves not only improving the quality and dignity of life, but also a considerable saving of money to the same family and the public health system. Suffice to say that taking care of a cancer sufferer to our Foundation costs about 2,500 Euros for 100 days, while a single day in the hospital for an admission costs alone over 600 Euro.

The effectiveness of care model of ANT Foundation is validated by the results of quality indicators. To detect the customer satisfaction, since 2009 ANT has formulated a questionnaire in order to assess the quality of service perceived by the caregiver. This is sent home via postal mail, about a month after the exitus of patients, to the family. In 2012, caregivers expressed their satisfaction for ANT services as high/very high in $95.7 \%$ of the cases.

Another important indicator of the quality of home care that supports ANT regards the place of death of patients. 79\% (national average ANT) of the assisted choose to die in their own homes. To the north of Italy, this percentage is $71 \%$ for the center is equal to $74.3 \%$ and for the South to $93 \%$. These data are of great importance if compared with the data concerning the place of death of cancer patients in Italy that indicate a national average of deaths at home $57.9 \%$ (varies widely from $28.2 \%$ in the Northeast to $94 \%$ in the south of the country).

The charitable model of ANT, like all models, may still be lacking, in the sense that it needs to be improved and expanded in order to assist more and more people and offer them more services. And to do this we must build solid paths of cooperation with other local, public and private, organizations, in order to achieve increasingly higher standard of care, while all of them maintaining their own specific areas. We strongly agree on the need to confront with specialists, structures and institutions dealing with cancer care and palliative care, both in Italy and in other countries, in order to share our experience and contribute to the creation of a more effective and efficient network of care.

The topics, from clinical issues to those most relevant organizational models, are numerous and we believe absolutely central as regards the present and especially the future of palliative care. Future must be built by all of us, together, in other words from everybody who has, and will have, the privilege of taking care of cancer patients and their families. 